\title{
Therapeutic Efficacy of Phyllanthus emblica L. Fruit Extract and Cisplatin Combination against 7, 12-dimethylbenz(a)anthracene Induced Hamster Buccal Pouch Carcinogenesis
}

\author{
S. Mirunalini* and M. Krishnaveni
}

Assistant Professor in Biochemistry \& Biotechnology, Annamalai University, Annamalainagar-608 002, Tamil Nadu, India; mirunasankar@gmail.com

\begin{abstract}
Amla fruit has been conventionally used as a health food for the prevention and therapy of cancer. Accordingly, the study aimed to explore the chemotherapeutic efficacy of amla and cisplatin combination on molecular mechanistic apoptosis implicated in proliferation, apoptosis and angiogenesis moleules during DMBA-induced buccal pouch carcinogenesis. Our western blot results showed that up regulation of Bcl-2, cyclin D1, Proliferating cell nuclear antigen, Cdc25C, Cox-2, Vascular endothelial growth factor and Survivin were observed in DMBA treated groups, whereas after treatment with combination therapy significantly down regulated the above noted proteins expression level. It was also observed that combination of amla and cisplatin treated tumor groups induced increased levels of Bax, caspase-3 and caspase-9 levels. More intriguingly, the effect was more pronounced in the tumor animals receiving combination therapy suggesting a synergistic effect. The results clearly suggest that combination therapy of amla and cisplatin had inhibitory effect during oral carcinogenesis via induction of apoptosis and suppression of molecules involved in cell proliferation and angiogenesis. Thus, amla fruit contains novel rich source of natural antioxidants and can be considered as an adjuvant therapeutic agent with cisplatin for oral cancer.
\end{abstract}

Keywords: Angiogenesis, Apoptosis, Buccal Pouch Cancer, Chemotherapy, Phyllanthus emblica, Proliferation

\section{Introduction}

Oral Squamous Cell Carcinoma (OSCC) is one of the most widespread malignant cancers of the oral cavity. The risk factors for oral cancer are alcohol use, betel quid chewing and tobacco chewing [1]. The incidence of OSCC is increasing among young patients less than 40 years old [6]. Various treatment modalities for oral cancer includes surgery, radiation, chemotherapy or a combination of surgery and radiotherapy, but such treatments are not effective and successful due to resistance of cancer cells against many chemotherapeutic agents cisplatin and paclitaxel [4]. Cisplatin is one of the most effective and widely used chemotherapeutic drugs, which exerts mitochondrial dysfunction and cytotoxicity in cancer cells [2]. Unfortunately, the main obstacles using cisplatin in clinical trials are toxic effects such as nephrotoxicity and neurotoxicity. Therefore, identification of novel therapeutic natural products that circumvent toxicities towards normal cells would have significant impact upon cisplatin based therapy. Thus, combination strategy may provide a more efficient therapeutic effect in various malignancies.

Over the recent decades various epidemiological studies have provided convincing evidence for the chemo 
preventive and therapeutic effects of natural food products. In this regard, one such natural source is amla that has received great attention owing to its wide pharmacological applications. Our previous studies have shown significant inhibition of buccal pouch cancer in the hamster model by amla fruit extract.

The present work focuses on elucidating the therapeutic effect of amla with cisplatin combination on DMBA-induced buccal pouch cancer model by analysing the expression of apoptotic, anti-apoptotic, proliferative and angiogenesis related proteins. As reported herein, our novel observations indicate that amla had synergistic apoptotic effect in buccal pouch cancer model, which may be an effective strategy to augment the therapeutic efficacy of cisplatin.

\section{Materials and Methods}

\subsection{Plant Material and Preparation of Amla Methanolic Fruit Extract}

Fresh fruits of Phyllanthus emblica were washed with water and dried in shade condition. 500g of dried fruit powder were soaked in $2 \mathrm{~L}$ methanol for 3 days. After 3 days, the extracts were filtered using muslin cloth and Whatman No:1 filter paper and the procedure were repeated thrice. Then, the filtrates were collected and evaporated on a rotary evaporator (Buchi Rotavapour, Switzerland) under reduced pressure. The yield of the extracts was $9 \%(\mathrm{w} / \mathrm{w})$ and stored at $4^{\circ} \mathrm{C}$. The total polyphenol, flavonoid and tannin contents of this extract were estimated.

\subsection{Selection of Animals and Feed Ratio}

6-10 weeks old male Syrian hamsters (weighed 80-150g) were purchased from National Institute of Nutrition (NIN), Hyderabad. All animals were housed in polypropylene cages under controlled environmental temperature with a $12 \mathrm{~h}$ light/dark cycle $\left(22 \pm 2^{\circ} \mathrm{C}\right)$ and relative humidity (55 $\pm 10 \%)$ and provided with free access to pellet food and water ad libitum. The standard pellet diet comprised of crude oil $(4.12 \%)$, crude fiber $(4.12 \%)$, crude protein $(22.12 \%)$, ash $(5.17 \%)$, and sand silica (1.13\%) with energy value of $3625 \mathrm{Kcal} / \mathrm{kg}$. Animal care and treatment was performed in accordance with the guidelines of the Indian National law on animal care and use and approved by the Institutional Animal ethical committee at faculty of Medicine,
Annamalai University (Reg.no.160/1999/CPCSEA, proposal no.732).

\subsection{Treatment Regimen}

The chemo preventive efficacy of Phyllanthus emblica fruit methanolic extract was investigated using a wellestablished 7,12-Dimethylbenz(a)anthracene (DMBA) induced buccal pouch carcinogenesis model in hamsters. In order to study the chemotherapeutic effects of amla and cisplatin, the animals were allocated into 6 groups of 6 animals each. The total experiment was conducted over period of 18 weeks.

Group 1 - Served as control group, which were treated with mineral oil only.

Group 2 - Received Phyllanthus emblica methanolic fruit extract (PFMet) $(200 \mathrm{mg} / \mathrm{kg} \mathrm{BW})$ thrice a week for a period of 18 weeks.

Group 3 - The left buccal pouches of animals in group 3 were painted with $0.5 \%$ DMBA in mineral oil using a no. 4 brush thrice a week for 10 weeks. Approximately, 0.2 $\mathrm{ml}$ of the DMBA solution was used in each application.

Group 4 -Tumor bearing hamsters in group 4 were given an oral administration of PFMet $(200 \mathrm{mg} / \mathrm{kg})$ alone thrice a week for 8 weeks starting from the 10 weeks of tumor formation.

Group 5 - Cancer bearing animals were administered with an intraperitoneal injection of Cis $(2 \mathrm{mg} / \mathrm{kg}$ BW) alone once a week for 8 weeks starting from the 10 weeks of Tumor formation.

Group 6 - Were treated with combinations of PFMet $(200 \mathrm{mg} / \mathrm{kg} \mathrm{BW})$ and Cis $(2 \mathrm{mg} / \mathrm{kg}$ BW $)$ as described in group 4 and group 5 .

The dose for amla and cisplatin used in this study were chosen on the basis of previous reports [5]. The experiment was terminated at the end of 18 weeks and animals were sacrificed by cervical decapitation. To scrutinize amla and cisplatin toxicity, the body weight of each animal was measured every week over 18 weeks.

\subsection{Western Blot Analysis}

Approximately $30 \mathrm{mg}$ of each tissues samples were washed thrice with PBS and lysed in cell lysis buffer (0.05 $\mathrm{mol} / \mathrm{l}$ Tris- $\mathrm{HCl}(\mathrm{pH} 7.5), 0.15 \mathrm{~mol} / \mathrm{l} \mathrm{NaCl}, 0.001 \mathrm{~mol} / \mathrm{l}$ PMSF, $0.001 \mathrm{~mol} / 1$ EDTA (pH 8.0), 1\%Triton X-100, $0.1 \%$ SDS, $2 \mu \mathrm{g} / \mathrm{ml}$ Leupeptin). The lysates were centrifuged at $12,000 \mathrm{~g}$ for $5 \mathrm{~min}$ at $4^{\circ} \mathrm{C}$. The protein extracts

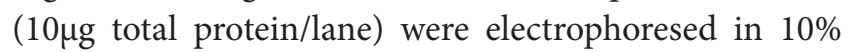


SDS polyacrylamide gel and then transferred on to polyvinylidene difluoride (PVDF) membranes. Membranes were blocked with $5 \%$ skimmed milk at room temperature for $1 \mathrm{~h}$ followed by incubation with the primary antibodies against anti-cyclin D1 (dilution 1:2000), mouse monoclonal primary antibodies against anti-human Caspase 3 (1:2000), anti-human Caspase 9 (1:2000), antihuman Bax (1:2000), anti-human Bcl-2 (1:2000), antihuman Cox-2, (1:2000), anti-human survivin (1:2000), anti-human VEGF $(1: 2000)$ at $37^{\circ} \mathrm{C}$ for 1 hour. After washing for 15 minute with Phosphate Buffered Saline (PBS), membranes were then incubated with the corresponding horseradish peroxidase conjugated goat antimouse secondary antibodies (dilution $1: 2000$ ) for $1 \mathrm{~h}$ at room temperature and then washed for 15 minutes with PBS. The proteins were visualized using the Enhanced Chemiluminesence (ECL) plus western blotting detection reagents.

\subsection{Buccal Pouch Histopathological Evaluation}

For, histopathology observation, portions of buccal, liver and kidney tissues of control, tumor and treated groups were fixed in 10\% formalin and stained with haematoxylin and eosin and examined under a microscope (H and $\mathrm{E} 40 \mathrm{x}$ ).

\subsection{Immunohistochemical Evaluation}

Tumor sections slides were de-paraffinized using xylene, rehydrated in graded ethanol for $15 \mathrm{~min}$ at room temperature. Antigen retrieval was done using citrate buffer, $\mathrm{pH} 6.0$ for $20 \mathrm{~min}$ and the tissues were washed with Tris-buffered saline (TBS). Endogenous peroxidase activity was inhibited by $3 \% \mathrm{H}_{2} \mathrm{O}_{2}$ in methanol. The sections were incubated with normal goat serum for 15 minutes to prevent non-specific antibody binding. The sections were then incubated with cytokeratin and $\mathrm{Ki}-67$ mouse monoclonal antibody at $4^{\circ} \mathrm{C}$. After washing with TBS, the sections were incubated with anti-mouse biotinylated secondary antibody (1:200) followed by streptavidin horse radish peroxidase $(1: 1000)$ for $10 \mathrm{~min}$ and the positive reactions were visualized with 3,3'-diaminobenzidine and counterstained with haemotoxylin. The primary antibody was omitted and substituted with the TBS served as negative controls. The proliferation index was calculated by Ki- 67 positive cells multiplied by 100 and then divided by number of cells.

\section{Results}

\subsection{Therapeutic Effects of PFMet and Cis Combination on Body Weight Changes during DMBA-Induced Buccal Pouch Carcinogenesis}

Figure 1 shows the changes in the body weight of hamsters in control and experimental groups. The body weight was significantly decreased in DMBA group compared to control group. However, PFMet, Cis and combination group showed a significant improvement in DMBA painted group. Importantly, the combination was more pronounced in improving the body weight than the individual agent alone. No statistical differences were noticed in body weight of hamsters between control and PFMet alone treated groups $(\mathrm{P}<0.05)$. No systemic toxic effects as evidenced in Cis and combination group throughout the experimental period this was evidenced by the results of body weight.

Our results clearly showed that the supplementation of amla and cisplatin in combination was found to be synergistically effective in improving the body weight, implying that the toxicity of treatments were not severe. Besides, in vivo toxicity was also examined from changes in the body weight. Moreover, no obvious side effects were observed by examining the histologic features of liver and kidney of the animals in the control and treated groups, signifying that amla acts as a chemotherapeutic agent.

\subsection{Therapeutic Effects of PFMet and Cis Combination on the Expression Levels of Bcl-2 and Bax Proteins on DMBA- Induced Oral Cancer}

The expression levels of $\mathrm{Bcl}-2$ and Bax in DMBAinduced buccal pouch cancer of control and experimental groups were shown in Figure 2. According to our results, animals in the DMBA group had significantly higher level of Bcl-2 and lower level of Bax than the animals in the control group. In response to PFMet, Cis, combination group treated tumor group significantly declined the level of Bcl-2 and augment the Bax expression level. Interestingly, clear synergistic effects observed in combination therapy group showed more up regulated Bax and down regulated Bcl-2 level. But there were no significant differences in the levels of $\mathrm{Bcl}-2$ and $\mathrm{Bax}$ expression between the groups treated with PFMet alone and control groups $(\mathrm{P}<0.05)$. 


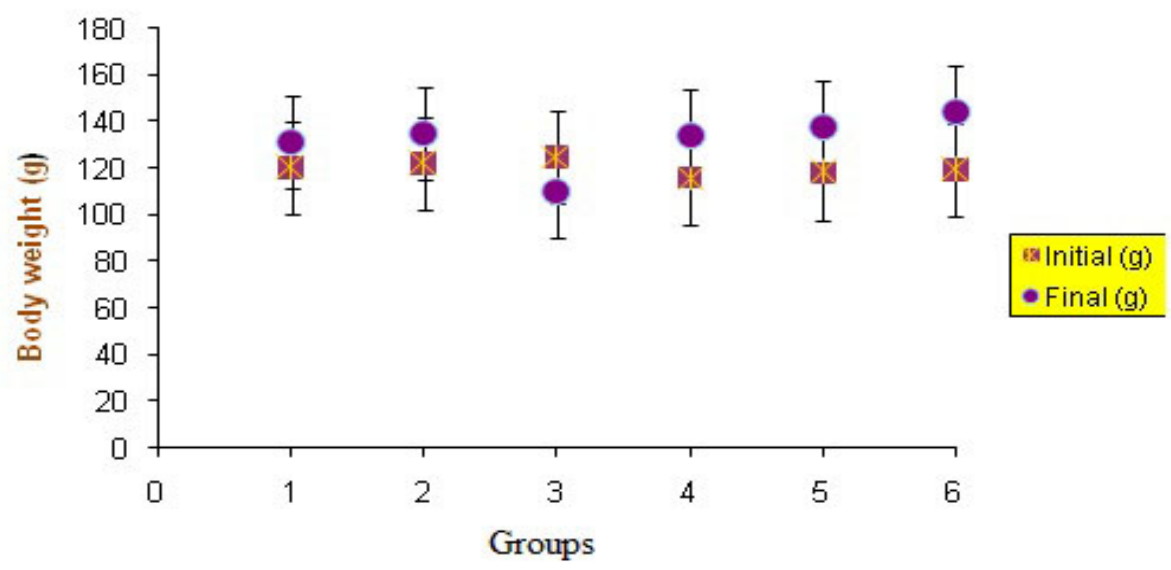

Figure 1. Combined effects of PFMet and Cis on body weight changes during DMBA-induced hamster buccal pouch cancer Results are expressed as the mean \pm S.D. $(n=6)$. Values not sharing a common superscript letter differ significantly at $\mathrm{P}<0.05$ (DMRT).

\section{Figure Caption}

Figures 1-5 depict the buccal pouch tissue of control and experimental animals by Western blotting. Lane 1. Control, Lane 2. Control + PFMet (200mg/kg BW), Lane 3. DMBA, Lane 4. DMBA + PFMet (200mg/kg BW), Lane 5. $\mathrm{DMBA}+\mathrm{Cis}(2 \mathrm{mg} / \mathrm{kg} \mathrm{BW})$, Lane 6. DMBA + PFMet + Cis. $\beta$-actin was used as an internal control. Data are representative of three independent experiments (mean \pm S.D). (B) The intensity of the protein bands was quantified by densitometer Histogram and the values were normalized to that of the control group and expressed as 1 unit. ${ }^{a} P<0.05$ compared with control groups.

${ }^{*},{ }^{*} P<0.05$ compared with DMBA group and amla and Cis individual agent treated groups.

\subsection{Therapeutic Effects of PFMet and Cis Combination on Apoptotic Proteins on DMBA-Induced Oral Cancer}

To determine the apoptosis induced by PFMet and Cis combination, the expressions of apoptotic proteins were detected by western blotting. As can be seen in Figure 3, the expressions of caspase 9 and caspase-3 were down regulated in DMBA treated group with respect to control group. Upon administration with PFMet, Cis and combination treated tumor group significantly up regulated the above noted proteins expression in comparison with DMBA group, which indicated that induction of apoptosis, is related to caspase- 9 activation. More intriguingly, the combination therapy achieved the synergistic up-regulation of caspase- 3 and caspase-9, in response to treatment with individual PFMet and cisplatin alone treated tumor group. Although, there was no statistical significant alterations of above mentioned proteins expressions level between PFMet alone and control groups $(\mathrm{P}<0.05)$
Apoptosis is a fundamental biological process which plays a pivotal role in the regulation of cancer development and progression. Neoplastic cells has the ability to propagate and circumvent apoptosis is a frequent features of human cancers, which represents a major target of anti-cancer therapeutic interventions [3].

Additionally, results demonstrated that combination therapy treated DMBA animals synergistically down-regulated Bcl-2 and up-regulated the expression of Bax, caspase-9, caspase-3, which lead to the activation of caspase cascades and subsequently induce apoptotic cell death. Based on this data, we conjecture that the observed apoptosis inducing effects by amla fruit extract may be due to the presence of synergistic interaction of bioactive compounds.

\subsection{Therapeutic Effects of PFMet and Cis Combination on Cell Cycle Related Proteins on DMBA-Induced Oral Cancer}

To gain insight into the mechanism of PFMet and Cis induced apoptosis, the protein expressions of cyclin 
A)

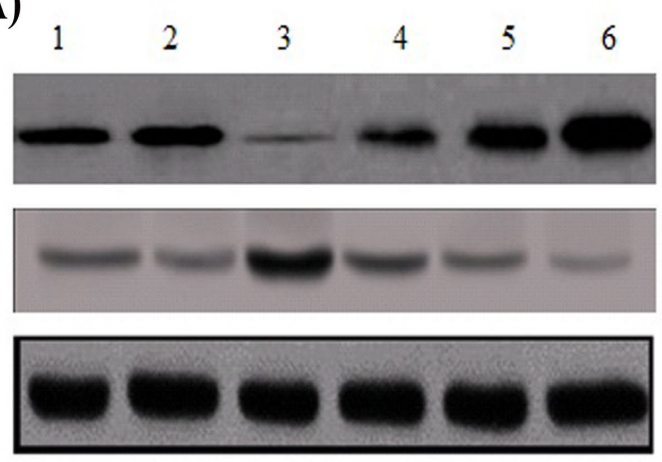

Bax

Bcl-2

$\beta$-Actin

\section{B)}

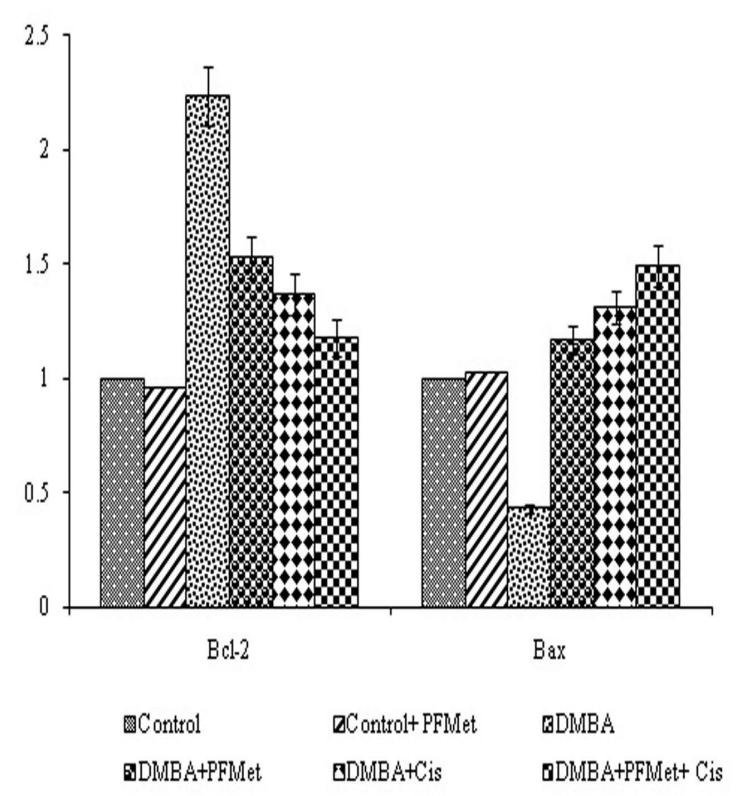

Figure 2. Therapeutic effects of PFMet and cisplatin combination on Bcl-2 and Bax protein expressions.

D1, Cdc25C, and PCNA were analysed by western blotting. As shown in Figure 4, we found that the hamsters receiving DMBA group showed higher levels of cyclin D1, Cdc25C, and PCNA than the animals in control group. Down regulation of cyclin D1, PCNA, and Cdc25C were observed in PFMet, Cis and the combination treated tumor group when compared with DMBA group. More intriguingly, the combination therapy was found to be effective in modulating the levels of above mentioned proteins expression. However, no significant changes were observed in the levels of proteins cyclin D1, Cdc25C, and PCNA between control and PFMet alone treated groups.
A)

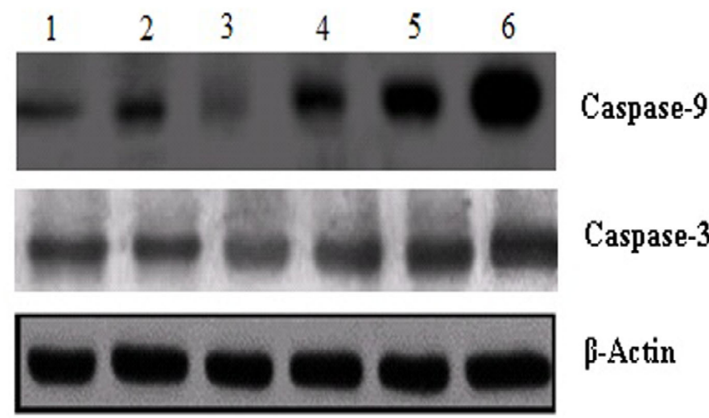

B)

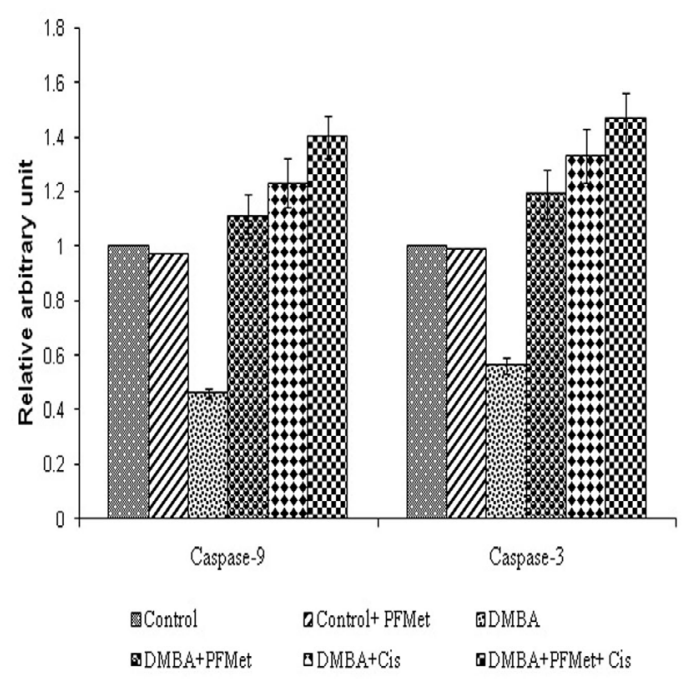

Figure 3. Therapeutic effects of PFMet and cisplatin combination on activation of caspase-3 and caspase-9 protein expressions.

\subsection{Therapeutic Effects of PFMet and Cis Combination on Cox-2, Survivin and VEGF Proteins on DMBA-Induced Oral Cancer}

To further confirm the anti-proliferative and antiangiogenic effect of PFMet and Cis combination, we then examined the expressions of Cox-2, survivin and VEGF using western blotting. As depicted in Figure 5, in comparison with control group, DMBA treated hamsters showed higher levels of Cox-2, VEGF and survivin, while in amla, Cis alone treated tumor group and combination group showed lower levels of above mentioned proteins. 
A)

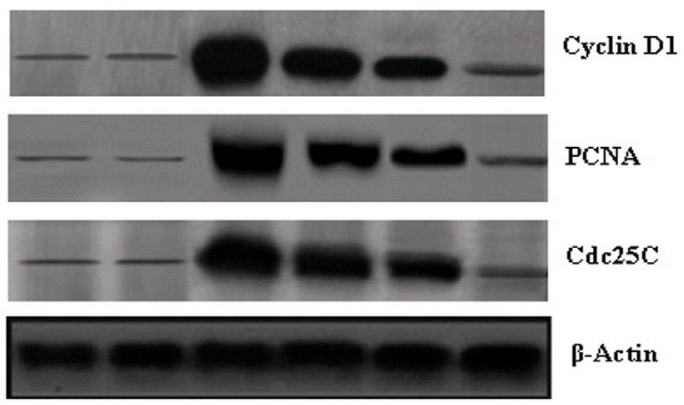

B)

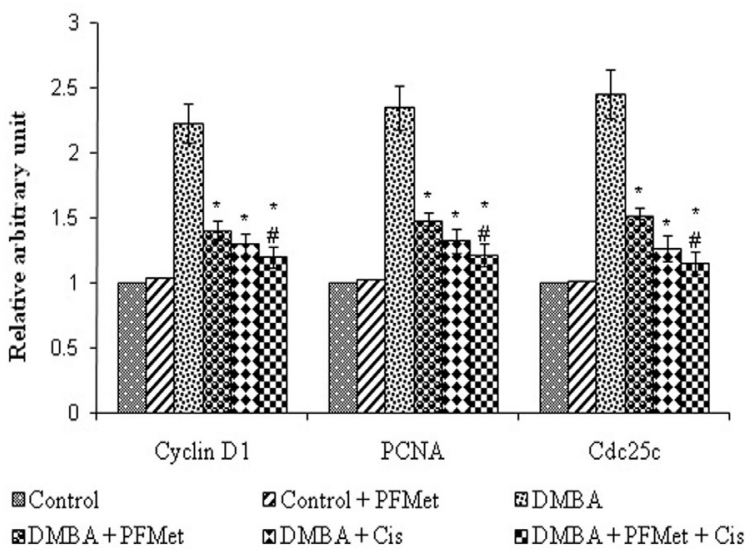

Figure 4. Therapeutic effects of PFMet and cisplatin combination on cell cycle related protein expressions.

Especially, the effect was more pronounced in the animals supplemented with combination therapy exhibited synergistically down regulated the levels of Cox-2, VEGF and survivin than the individual agent PFMet or Cis alone treated DMBA group. Although, there were no statistical significant alterations in Cox-2, VEGF and survivin expression levels among PFMet alone and control group $(\mathrm{P}<0.05)$.

\subsection{Therapeutic Effects of PFMet and Cis Combination on Buccal Pouch, Liver and Kidney Histochemical Analysis}

Histopathological findings on buccal pouch sections from different groups of control and experimental animals are illustrated in Figure 6. The buccal pouch sections of control hamsters group showed normal architecture epithelium. PFMet alone supplemented group revealed normal architecture. Hamsters received DMBA exhibited well differentiated squamous cell carcinoma around formation of keratin pearls. Tumor hamsters supplemented with PFMet and Cis showed severe and
A)

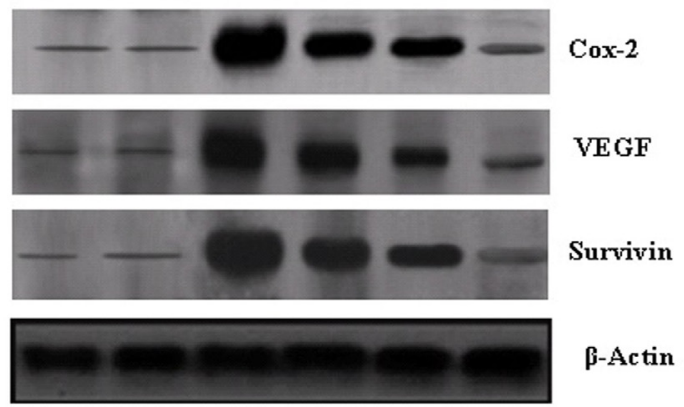

B)

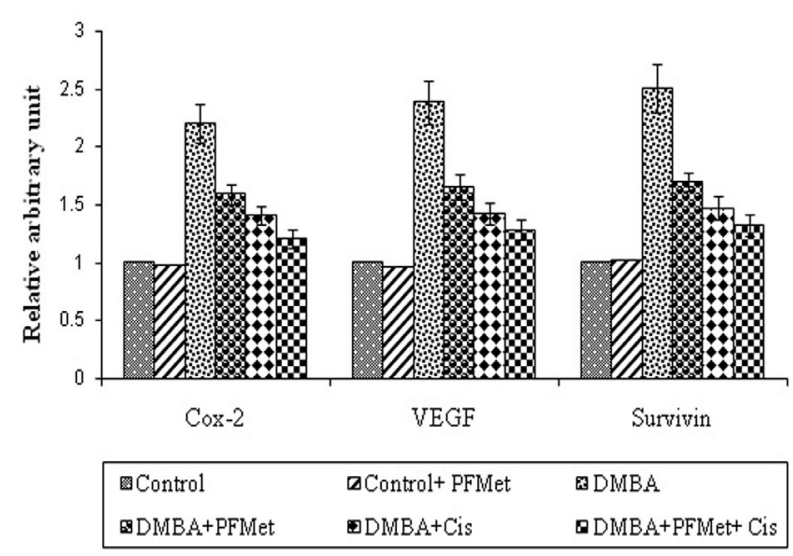

Figure 5. Therapeutic effects of PFMet and cisplatin combination on Cox-2, VEGF and survivin protein expressions. moderate dysplasia. Combination therapy of amla and Cis to DMBA treated animals showed severe hyperplasia.

In order to evaluate adverse effects of combination therapy, histopathological studies were performed on liver and kidney tissues of control, tumor and PFMet and Cis combination treated animals (Figure 7). Liver and kidney tissues from cancer bearing hamsters showed infiltration of inflammatory cells and loss of glomerulus, after treatment with combination therapy revealed normal morphology with limited infiltration in hepatocytes and reduced swelling of tubules. Consequently, our histo evaluation results propose that supplementation with amla fruit extract in combination with cisplatin did not affect the morphology of liver and kidney organs.

\subsection{Therapeutic Effects of PFMet and Cis Combination on Immunocytokeratin Analysis}

The antitumor effects of amla were evaluated on the basis of immunohistochemical analysis of tumors dis- 

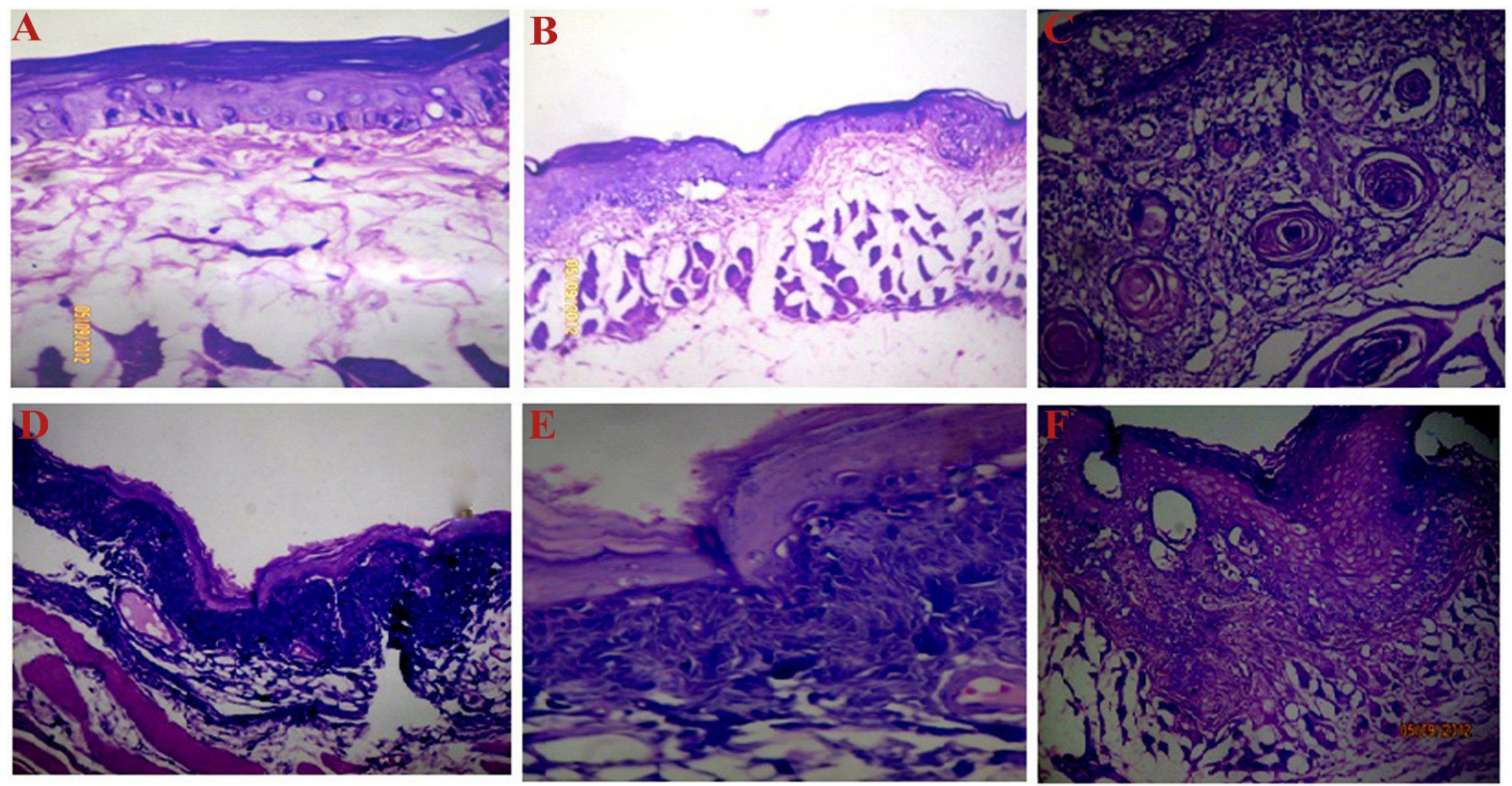

Figure 6. Representative histopathological alterations in buccal tissues of control and DMBA treated groups. (A) Control hamsters showing normal squamous epithelium. (B) PFMet alone supplemented group showing normal epithelium. (C) Hamsters received DMBA group showing well differentiated carcinoma around formation of keratin pearls. (D) Tumor hamsters supplemented with PFMet and Cis showing severe and moderate dysplasia. (E) Combination therapy of amla and Cis to DMBA treated animals showing severe hyperplasia.
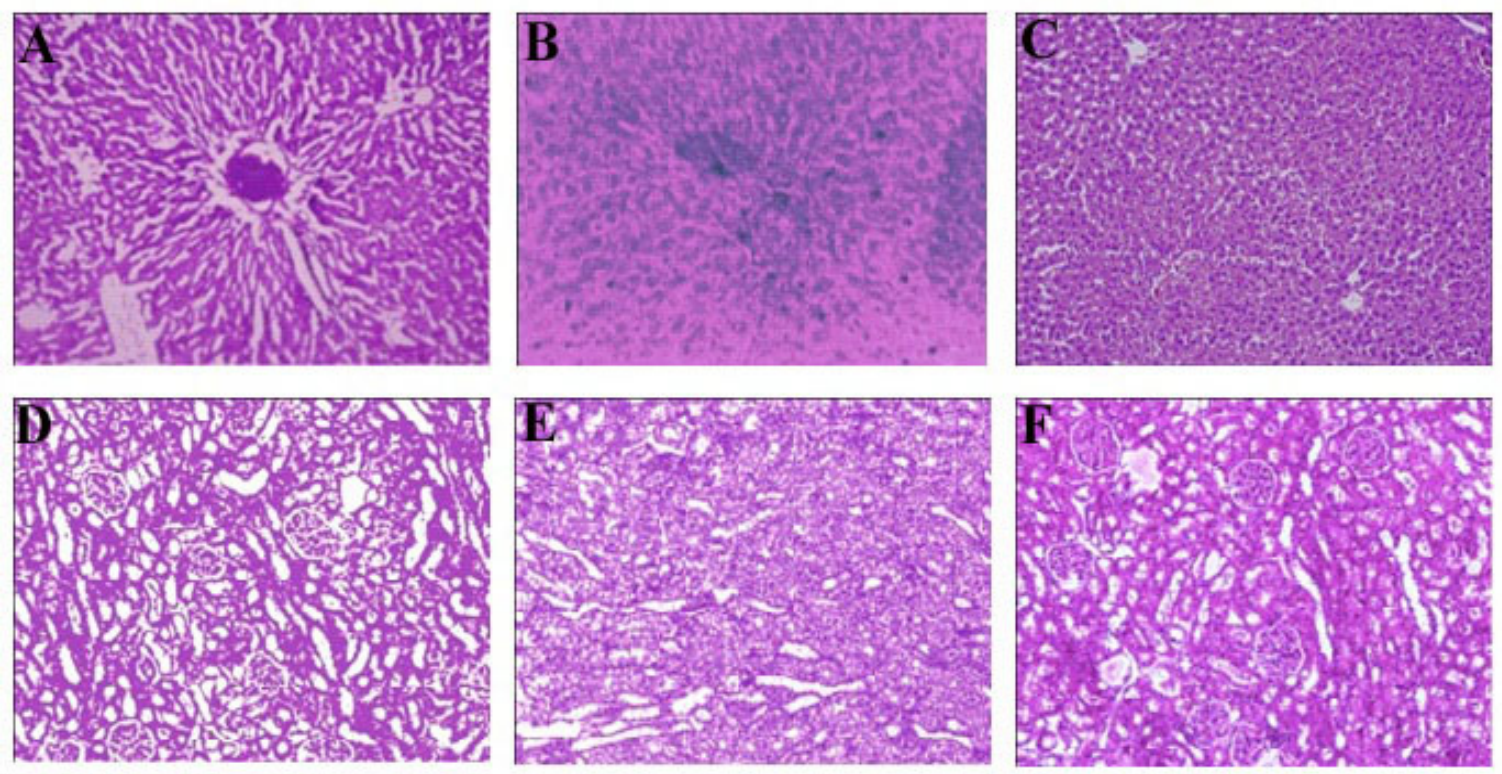

Figure 7. No adverse effects observed in combination therapy treated groups. Hematoxylin and eosin staining of liver and kidney from the control, tumor and combination therapy treated groups. (A) Control hamsters showing normal central vein. (B) DMBA hamsters groups showing microvesicular fatty changes and inflammatory cell infiltration. (C) Combination therapy treated groups showing normal cells with reduction in fatty changes. (D) Hematoxylin and eosin staining of kidney from the control, tumor and combination therapy treated groups. Control hamsters showing normal glomeruli and tubules. (E) DMBA treated hamsters groups showing swelling of tubules with abnormal morphology. (F) Combination therapy treated groups showing normal architecture with reduced swelling of tubules. 

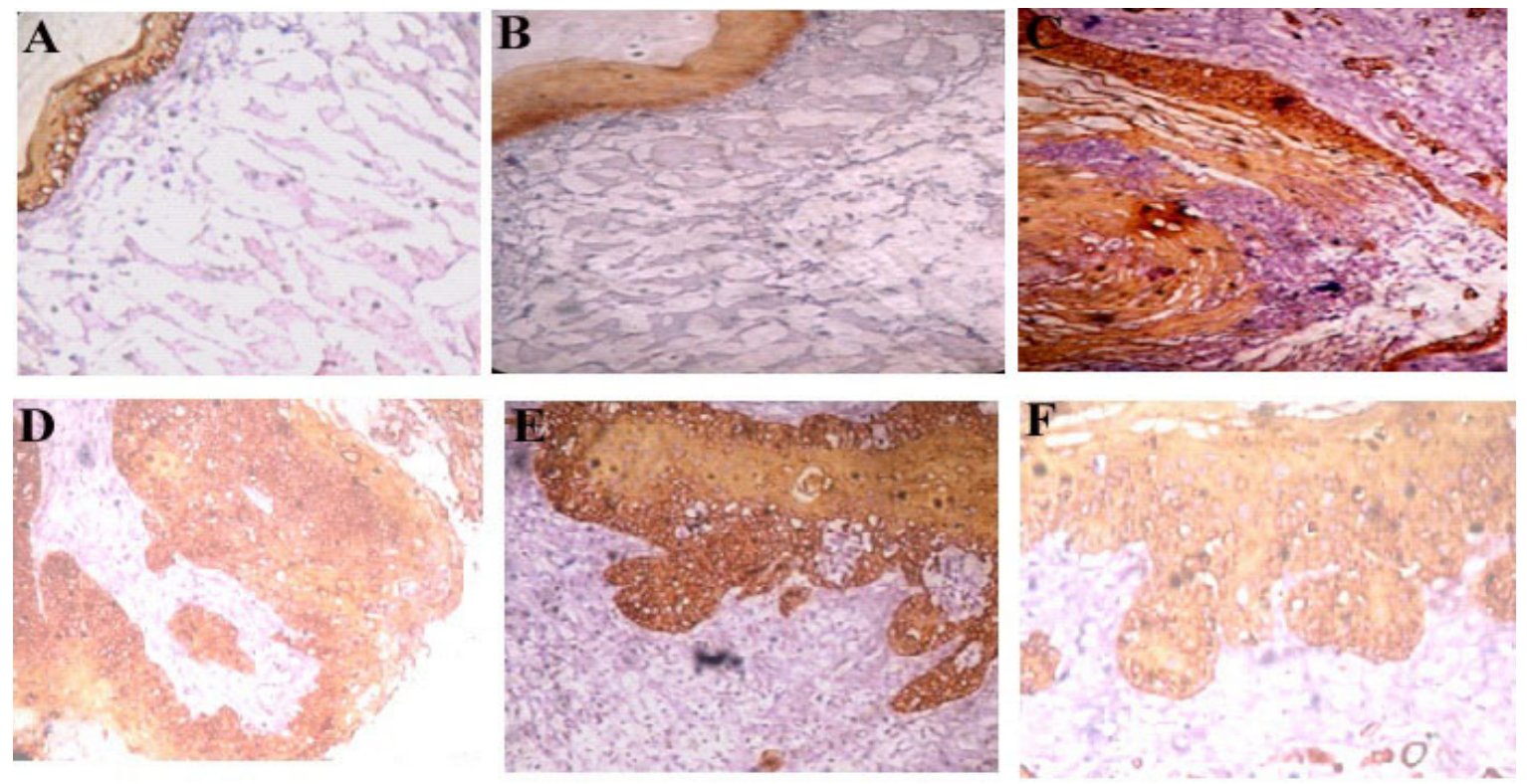

Figure 8. Combination therapy of PFMet and cisplatin on Immunohistochemical cytokeratin 8 alterations in buccal tissues of control and DMBA treated groups. (A) Control group shows negative staining in epithelial layer. (B) PFMet alone treated group shows negative staining in epithelial layer. (C) DMBA group shows well differentiated carcinoma with increased positive staining. Groups D, E and F show reduction in expression.

sected from control, amla, cisplatin, and combinationtreated animals. Representative staining of immunocytokeratin expression profiles are depicted in Figure 8. The control and PFMet alone treated hamsters groups showed a negative staining of cytokeratin, while the DMBA group exhibited squamous cell carcinoma showed high expression of cytokeratin indicates positive stained cells, indicating abnormal cell growth during DMBA-induced oral lesions. Supplementation with PFMet, Cis and combination therapy treated groups hamsters revealed decreased expression of cytokeratin, when compared with DMBA group. Nevertheless, a maximum reduction was observed in the combination group compared to individual PFMet or Cis treated tumor group alone. There was no statistic difference in cytokeratin 8 expression between the PFMet alone and the control group.

\subsection{Therapeutic Effects of PFMet and Cis Combination on Ki-67 Analysis}

DMBA group had a stronger expression positive index of Ki-67 (11\%) in comparison with control group (Figure 9). After supplementation with combination therapy showed reduced expression of Ki-67 (3\%), which suggested that inhibitory effect of amla might be due to reduced cell proliferation. There was no statistic difference in Ki-67 expression between the PFMet alone and the control group.

Taken together, these results signify that above noted synergistic activity of combined bioactive phytochemicals present in amla fruit extract may reduce the risk of oral cancer possibly by suppressing inflammatory responses during DMBA carcinogenesis. Our results suggest that a probable molecular mechanism implicated in the anticancer activity of amla fruit extract is mediated via its anti-inflammatory action.

Amla fruit can significantly attenuate the efficacy of cisplatin anticancer activity in hamster buccal pouch model via inactivation of Bcl-2, cyclin D1, PCNA, Cdc25c, Cox-2, VEGF and survivin proteins and activation of Bax, caspase- 9 and caspase- 3 proteins. The histopathological evaluation showed that morphology of the liver and kidney tissues was unaltered by the amla in combination with cisplatin treated groups. The data from the immunohistochemical staining of Ki67 and cytokeratin tumor cell growth was significantly suppressed in animals treated with combination therapy treated group. Our immunohisto data showed that amla exerts apoptotic effect and enhanced the cytotoxicity of cisplatin through the down regulation of cytokeratin and showed antiproliferative effect via 

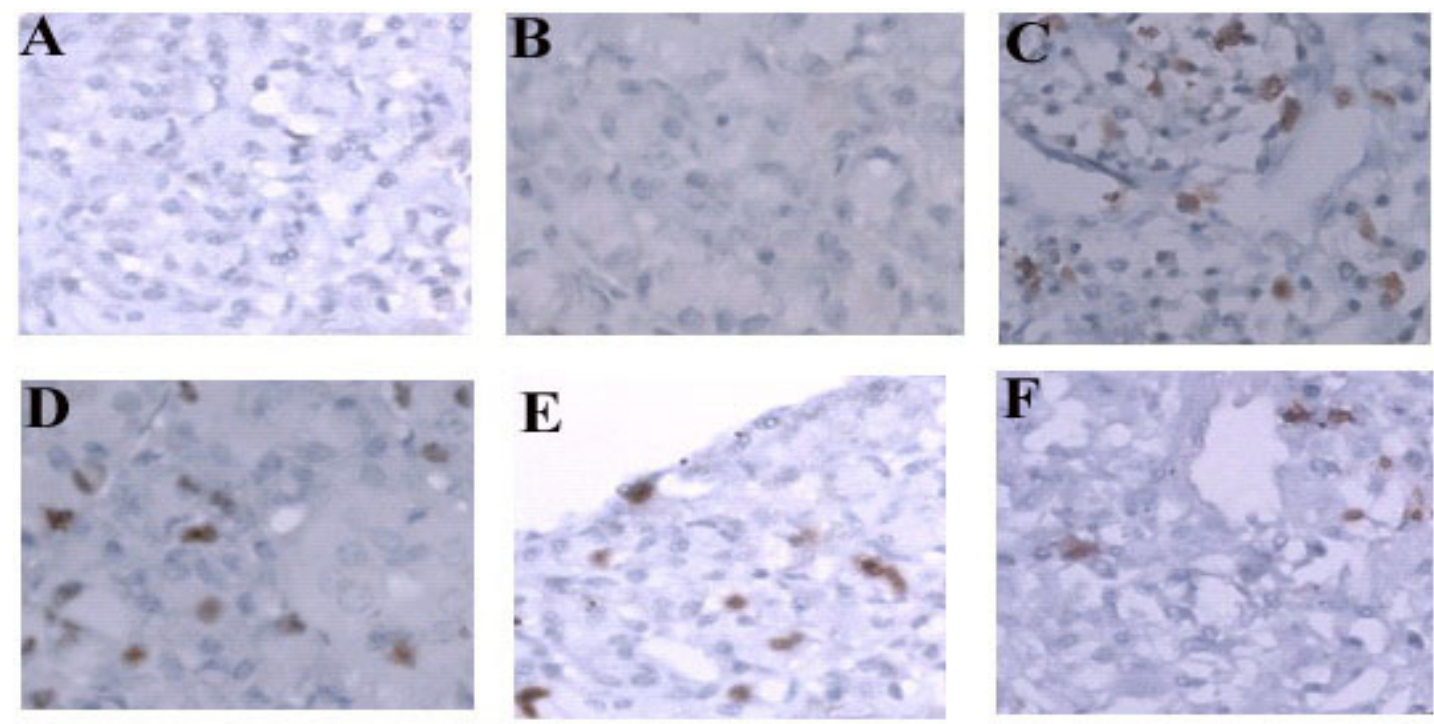

Figure 9. Combination therapy of PFMet and cisplatin on Immunohisto Ki-67 proliferation expression in buccal tissues of control and DMBA treated groups. (A) Control group showing negative staining. (B) PFMet alone treated group showing negative staining. (C) DMBA group showing increased positive staining in nuclear cells. (D) DMBA + PFMet group showing reduced positive staining in nuclear cells (E) DMBA + Cisplatin group showing lowered positive stained nuclear cells. (F) $\mathrm{DMBA}+\mathrm{PFMet}+\mathrm{Cis}$ group showing negative staining. Arrows indicate the positive stained PCNA cells.

down regulation of Ki67 secretion in hamster buccal pouch carcinogenesis model. Thus, the present study emphasizes the high therapeutic potential of amla. The antitumor activities of amla fruit extract demonstrated a synergistic effect with significant antitumor effect on DMBA-induced hamster buccal pouch carcinogenesis model.

\section{Conclusion}

These findings indicate for the first time that the combination of amla/cis displayed a better treatment modality for inhibition of oral carcinogenesis by stimulating apoptosis and abrogating anti-apoptotic, proliferative, angiogenic proteins. The precise molecular mechanisms underlying amla and cisplatin induced apoptosis have not yet been investigated. Therefore, amla can ameliorate the side effects of cisplatin towards non-specific normal tissues. In this sense, amla can be used as dietary supplemental health promoting agents in functional foods and recommended as an anticancer adjuvant agent among cancer patients. Further study warrants the isolation of bioactive compounds from the amla fruit extract and to study the interaction of active compounds with cisplatin combination to target the various signaling pathways involved in oral cancer.

\section{References}

1. Ko Y. C., Huang Y. L., Lee C. H., Chen M. J., Lin L. M., and Tsai C. C., "Betel quid a chewing, cigarette smoking and alcohol consumption related to oral cancer in Taiwan", J Oral Pathol Med, vol. 24, p. 450-453, 1995.

2. Meijer C., Van Luyn M. J., Nienhuis E. F., Blom N., Mulder N. H., and De Vries E. G., "Ultrastructural morphology and localization of cisplatin-induced platinum-DNA adducts in a cisplatin-sensitive and -resistant human small cell lung cancer cell line using electron microscopy", Biochem Pharmacol, vol. 61, p. 573-578, 2001.

3. Singh B. N., Fu J., Srivastava R. K., and Shankar S., "Hedgehog signaling antagonist GDC-(Vismodegib) inhibits pancreatic cancer stem cell characteristics: molecular mechanisms", PLoS One, vol. 6, e27306, 2011.

4. Wang D., and Lippard S. J., "Cellular processing of platinum anticancer drugs", Nat Rev Drug Disc, vol. 4, p. 307320, 2005.

5. Zhang Y., Wang C., Wang H., Wang K., Du Y., and Zhang J., "Combination of Tetrandrine with cisplatin enhances cytotoxicity through growth suppression and apoptosis in ovarian cancer in vitro and in vivo", Cancer Lett, vol. 304, p. 21-32, 2011.

6. Zygogianni A. G., Kyrgias G., Karakitsos P., Psyrri A., Kouvaris J., and Kelekis N., "Oral squamous cell cancer: early detection and the role of alcohol and smoking", Head Neck Oncol, vol. 3, p. 2, 2011. 